\title{
Ultrasound diagnosis of a pathological fracture of the phalanx in a patient with chronic kidney disease: After a 2-month diagnostic delay
}

\author{
Selcuk Sayilir \\ Department of Physical Medicine \& Rehabilitation, Mugla Sıtkı Kocman University Faculty of Medicine, Mugla, Turkey \\ Cite this article as: Sayilir S. Ultrasound diagnosis of a pathological fracture of the phalanx in a patient with chronic kidney disease: After \\ a 2-month diagnostic delay. North Clin Istanb 2018;5(2):144.
}

A 56-year-old man was admitted to our clinic with pain, swelling, and tenderness in the third finger of his right hand for 2 months. He did not report a physical injury. He was admitted to other clinics for these symptoms, where he was misdiagnosed inflammatory arthritis and treated with 'deflazacort ( $30 \mathrm{mg} /$ day). He has a history of chronic kidney disease (CKD) and has been undergoing hemodialysis for 4 years. On physical examination, he had pain and edema on the proximal interphalangeal joint of the right hand (Fig. 1), and no warmth or change in color was noted. In addition, there were no findings of active peripheral arthritis. Blood culture was positive for Staphylococcus epidermidis, and he was administered an intravenous antibiotic. An ultrasound examination performed for the finger due to these clinical findings showed a fracture line in the proximal phalanx of the third finger.

Peripheral and axial skeletal fractures are frequent complications of CKD as a result of altered mineral and bone metabolism and enhanced bone fragility. Several authors have reported that fractures can be considered dramatic events in CKD patients. The effectiveness of ultrasound examination in fracture diagnosis has also been reported in the literature. It was remarkable that the patient was admitted to different clinics for his complaints, but he was mistreated with corticosteroids and analgesics because of misdiagnosis with inflammatory arthritis. X-ray examination after the ultrasound examination also showed a fracture line, but it was not a clear view and may overlook (Fig. 1). If patients with immunodeficiency are inappropriately treated with corticosteroids, they can become susceptible to severe infections. The reported patient had a history of using corticosteroids for 2 months. He had a serious blood infection and was treated with intravenous antibiotics.
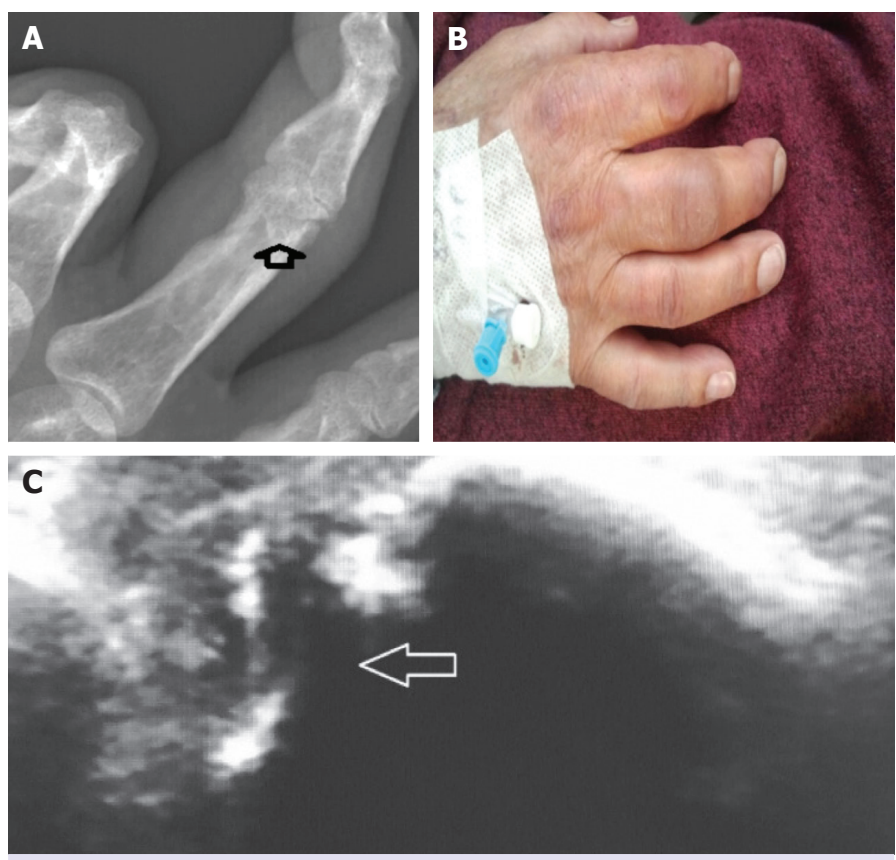

FIGURE 1. (A) X-ray showing a nebulous fracture line at the third proximal phalanx (arrow). (B) Edema in the third finger. (C) Ultrasound examination showed a fracture line at the proximal phalanx (black arrow)

Received: February 14, 2017 Accepted: July 27, 2017 Online: March 26, 2018

Correspondence: Dr. Selcuk SAYILIR. Department of Physical Medicine \& Rehabilitation, Mugla Sıtkı Kocman University Faculty of Medicine, Mugla, Turkey.

Phone: +90252 2141326 e-mail: selcukssay@gmail.com

(c) Copyright 2018 by Istanbul Provincial Directorate of Health - Available online at www.northclinist.com 